\title{
Coinfection of SFTSV Genotype A with Rickettsia among Farmers in Northeast
}

\author{
Yue Ma ${ }^{1}$, Hongyan $\mathrm{Liu}^{1}$, Jixu $\mathrm{Li}^{1}$, Dan $\mathrm{Li}^{1}$, Yan Wang${ }^{1}$, Fenghua Jiang${ }^{1}$, Qingtian Gao ${ }^{1}$, \\ Xiaohu Han ${ }^{2}$, Guoshun Shen ${ }^{1}$, and Zeliang Chen ${ }^{2}$ \\ ${ }^{1}$ Affiliation not available \\ ${ }^{2}$ Shenyang Agricultural University
}

September 21, 2020

\begin{abstract}
Severe fever with thrombocytopenia syndrome (SFTS), caused by the SFTS virus (SFTSV), is a new acute infectious disease transmitted by ticks. Rickettsiosis is caused by Rickettsia spp. carried by ticks. Here, we conducted case analyses and molecular biological diagnosis of some patients with suspected SFTS in Liaoning Province in 2019 to identify rates of SFTSV and Rickettsia coinfection. Molecular diagnostic results confirmed SFTSV infection in 5 out of 17 suspected SFTS patients. Two strains of genotype A SFTSV were successfully isolated from patient blood. Ticks collected from the patient's locations had high positivity rates for SFTSV, which was highly similar to SFTSV from patients. Antibody testing of 7 patient serum samples revealed all samples to be positive for Rickettsia antibodies. These results indicate coinfection of SFTSV with Rickettsia that may complicate disease presentation and diagnosis. Therefore, coinfection of tick-borne disease should be considered for correct diagnosis and treatment.
\end{abstract}

\section{Introduction}

Severe fever with thrombocytopenia syndrome (SFTS) is a new acute infectious disease caused by the severe fever with thrombocytopenia syndrome virus (SFTSV) (Yu et al., 2011)belonging to a a novel phlebovirus (SFTS virus, SFTSV) in the family Phenuiviridae of the order Bunyavirales (Li et al., 2019). It was first reported in 2009 in the rural areas of the Hubei and Henan Provinces in Central China, and the population is generally susceptible. Since then, the incidence of SFTS has been successively reported in more than 10 provinces and cities in China(Zhang et al., 2013), and its epidemic area has a tendency to spread. The disease is also widely distributed in South Korea (Denic et al., 2011; Yun et al., 2016) and Japan(Takahashi et al., 2014), and cases of SFTS have also been reported in the United States (McMullan et al., 2012; Muehlenbachs et al., 2014). The main clinical manifestations of the disease are high fever, anorexia, muscle pain, chills, lymphadenopathy, leukopenia, thrombocytopenia, and multiple organ failure (Sun et al., 2019). Severe cases can lead to death.

SFTSV is an arbovirus mainly carried by ticks(Liu et al., 2012). The SFTSV virus genome is composed of three independent gene segments: L segment (6,368 bp; encodes the RNA-dependent RNA polymerase), $\mathrm{M}$ segment (3,378 bp; encodes the membrane protein precursor), and the ambisense $\mathrm{S}$ segment (1,744 bp; encodes the non-structural S protein and nucleocapsid protein)(Sun et al., 2014).

Rickettsiosis is a zoonotic disease mainly spread by ticks and is considered an important global emerging zoonotic disease (Parola, Paddock, \& Raoult, 2005). Humans are infected by the bite of ticks that carry rickettsial bacteria. Due to human factors such as lifestyle changes and population rates, and to environmental factors such as climate change, people have become more exposed to ticks. Rickettsiosis in humans usually refer to infections caused by bacterial species belonging to the genus Rickettsia and Orientia. It can 
be classified into typhus, spotted fever, and tsutsugamushi disease(Adem, 2019). Clinically, human serum rickettsia antibodies are used to determine whether the human body is infected withRickettsia. The typical clinical features of these diseases include: fever, fatigue, anorexia, nausea, headache, and rash, and the bite of the tick has eschar formation (Liu et al., 2016). These symptoms usually appear within 1-2 weeks after infection(Liu et al., 2016). The clinical manifestations of typhus and SFTS are similar, thereby complicating diagnosis.

In 2019, our laboratory carried out clinical analysis, laboratory diagnosis, and traceability studies of SFTSV in suspected cases in the Infectious Disease Hospital of Shenyang City to characterize SFTSV in patients in Northeast China. Molecular diagnosis and antibody testing for Rickettsia were also performed to determine the rates ofRickettsia coinfection with other tick-borne diseases, especially with SFTSV. The results we present here provide insights into the characteristics of SFTSV in Northeast China. Further, our findings can guide clinicians in the diagnosis of tick-borne diseases.

\section{Materials and Methods}

\subsection{Specimen source}

Blood sample: A total of 17 cases of suspected SFTS were reported from May 2019 to October 2019 in Shenyang Infectious Disease Hospital. Serum was collected from the patients and stored at $-80{ }^{\circ} \mathrm{C}$. human samples were reviewed and approved by the ethics committee of China CDC.

Tick samples: free ticks in patients' farmlands, forests, and other environments in nearby counties and cities were collected. Liquid nitrogen milling, and centrifugation were performed to prepare tick suspensions. Total nucleic acid extraction was performed as in the following method, and reverse screening for virus isolation was performed.

\subsection{Data collection}

After numbering all suspected SFTS patients, the patient's epidemiological history, systemic symptoms, neurological symptoms, respiratory symptoms, etc. are collected and classified by consulting medical records; at the same time, WBC, PLT, AST, ALT, CK, LDH, BUN and other laboratory inspection results are recorded.

\subsection{Nucleic acid extraction and reverse transcription}

TransGen's EasyPure Viral DNA/RNA Kit (Cat No: ER201-01) was used, and total nucleic acid was extracted according to the kit instructions. Vazyme Biotech's HiScript II Q RT SuperMix for qPCR (+gDNA wiper) (Cat No: R223-01) was used for reverse transcription according to the reagent instructions.

\subsection{SFTSV nucleic acid detection}

Using highly conserved regions in SFTSV $M$ as target regions, specific primers were designed for PCR amplification. The primers were MF3 (5'-GATGAGATGGTCCATGCTGATTCT-3') and MR2 (5'CTCATGGGGTGGAATGTCCTCAC-3') with a target band size of 560 bp. Amplification conditions: 94 , 5 min; 94,30 s, 5530 s, 721 min, 35 cycles; 72,5 min; 30- $\mu$ l system.

\subsection{Virus isolation}

The supernatant of Vero cells (African green monkey kidney cells) grown into a single layer was discarded, washed once with PBS, and incubated with $500 \mu \mathrm{L}$ of patient serum for $2 \mathrm{~h}$, and then discarded. Virus maintenance solution (DMEM containing $2 \%$ fetal bovine serum) was added and cultured at $37{ }^{\circ} \mathrm{C}$ under $5 \% \mathrm{CO}_{2}$, and cytopathic effects (CPEs) were observed daily. Tick suspensions that were positive SFTSV by the nucleic acid test were filtered through a $0.22-\mu \mathrm{m}$ bacterial filter and were added to the Vero cells. Cells were collected, RNA was extracted, reverse transcription was performed, and PCR detection was performed to verify successful infection. The infected cells positive for SFTSV were stored at $-80{ }^{\circ} \mathrm{C}$ until use.

\subsection{Amplification of SFTSV whole genome}


Previously designed primers for amplification of S (3 primer sets), M (7 primer sets), and L (13 primer sets) segments were used for whole genome sequencing. Primers were synthesized by Sangon Biotech (Shanghai) Co., Ltd. The size of the target band amplified by each primer set for the $\mathrm{S}$ and $\mathrm{M}$ fragments was around 600 bp. Amplification conditions: 94 , 5 min; 35 cycles: $94,30 \mathrm{~s} ; 55,30 \mathrm{~s} ; 721 \mathrm{~min} ; 72,5 \mathrm{~min} ; 30-\mu \mathrm{L}$ system. The size of the target band amplified by each primer set for the L fragment was about 350-650 bp. Amplification conditions: $94,5 \mathrm{~min} ; 35$ cycles: $94,30 \mathrm{~s} ; 63,30 \mathrm{~s} ; 72,1 \mathrm{~min} ; 72,5 \mathrm{~min} ; 30-\mu \mathrm{L}$ system.

\subsection{SFTSV whole genome sequencing and analysis}

The PCR products were subjected to agarose gel electrophoresis, and the amplified products were sent to Sangon Biotech (Shanghai) Co., Ltd for sequencing. The sequences were spliced using the SeqMan software. GenBank was used as source of reported strain sequences, and DNASTAR and MEGA6.0 were used to conduct a comprehensive analysis of the three fragments of the SFTSV strain, to identify the virus genotype, and to analyze the genetic evolution of the strain. For paired and multiple alignments, the maximum likelihood method (ML) was used to build a phylogenetic tree based on the L, M, and S sequences. The bootstrap method with 1000 replications was used to estimate the reliability of the branches.

\subsection{Molecular identification of Rickettsia}

The amplification and sequence analysis of the $16 \mathrm{~S}$, glt $A$, omp $A$, and ompB conserved sequences of Rickettsiacarried by ticks were performed based on the study published by $\mathrm{Xu}$ et al(Xu et al., 2019).

\subsection{Human serum Rickettsia-specific IgG antibody detection}

The human Rickettsia-IgG ELISA KIT (Cat No: JL48935) of Jianglai Biotech Co., Ltd. was used for IgG antibody detection and the detection antibody type was Rickettsia IgG. Serum from healthy people and distilled water were used as negative and blank controls, respectively.

First, The concentration of the standard product is $100,50,25,12.5,6.25,0 \mathrm{ng} / \mathrm{mL}$ in sequence, and 50 $\mu \mathrm{L}$ for each well. Next, Add sample diluent $40 \mu \mathrm{L}$ on the Enzyme labeling plate first, and then add the sample to be tested $10 \mu \mathrm{L}$ (the final dilution of the sample is 5 times). Add $100 \mu \mathrm{L}$ enzyme reagents to each well, except for blank wells. The samples were incubated at 37 for 1 hour and then washed with washing solution,repeat 5 times,pat dry. Add color developer A $50 \mu \mathrm{L}$ to each well, and then add color developer B $50 \mu \mathrm{L}$. Gently shake and mix well, and develop color at 37 in the dark for 15 minutes. Add stop solution 50 $\mu \mathrm{L}$ to each well to stop the reaction. $450 \mathrm{~nm}$ wavelength measurement of absorbance of each hole, OD was measured after zero-adjusting the blank control reaction.

\section{Results}

\subsection{Epidemiological information}

A total of 17 patients with suspected SFTS were admitted to the clinic (Table 1). Through molecular diagnostic tests, 5 patients were confirmed to have SFTSV infection. There were 2 males and 3 females, with an average age of 51 years. All of them are farmers, mainly farming in forest areas, and have history of animal contact (rats) or tick bites.

\subsection{Clinical manifestations}

Clinical examination revealed that SFTS patients developed fever, gastrointestinal symptoms, and neurological symptoms, which mostly included fever, headache, dizziness, body aches, nausea, vomiting, and fatigue. Among the symptoms, fever (17/17), muscle pain (17/17), and headache (16/17) were the most frequent symptoms of SFTS. Clinical manifestations in confirmed and suspected SFTS cases were similar, showing that clinical manifestations alone cannot be used for diagnosis of SFTS (Table 1).

\subsection{Laboratory test results}

The 5 patients diagnosed with SFTS mainly showed decreased white blood cell counts (WBC; 4/5), platelet counts (PLT; 5/5), alanine aminotransferase (ALT; 5/5), and aspartate aminotransferase (AST; 4/5), blood 
urea nitrogen (BUN; 2/5), creatine kinase (CK; 4/5), and increased lactate dehydrogenase (LDH; 5/5). All 5 patients with SFTS had thrombocytopenia, but other patients also had cytopenia (7/12) (Table 1).

\subsection{SFTSV and Rickettsia nucleic acid detection and virus isolation results}

From the 5 confirmed cases of SFTSV, 2 SFTSV strains were successfully isolated and named LNHDD20199 (from Dandong City, Liaoning Province) and LNHDG2019-6 (from Donggang City, Liaoning Province). NoRickettsia nucleic acid was detected in the serum or blood cells of the 17 patients suspected of SFTSV.

A total of 492 tick samples were collected from the patient's residence and surrounding areas (including Dandong City and, under its jurisdiction, Fengcheng City, Donggang City; Kuandian Manchu Autonomous County; and Zhuanghe City under the jurisdiciton of Dalian,which are all Changbai Mountain forest areas in the border area between China and North Korea). Environmental free tick samples (samples not collected in Beipiao City) were all identified by molecular biology. Among them, 12 samples were positive for SFTSV nucleic acids, with a positivity rate of $2.4 \%$. Two SFTSV strains were successfully isolated from tick samples and were named LNTEDG2019-6 (collected from Erdaogou, Dandong City, Liaoning Province) and LNTYJ2019-6 (collected from Yanji City, Jilin Province) (Figure 1).

\subsection{Amplification results of SFTSV whole genome sequence}

The L, M, and S fragments of four SFTSV strains were amplified separately, and the corresponding fragments were spliced to assemble the whole genome sequence. The sequences of SFTSV (LNHDD20199/Human/China/2019 in the evolution tree) S, M, and L fragments isolated from patient NO. 1 in Dandong has been uploaded to NCBI with accession numbers MT232960, MT232961, and MT232962, respectively.

\subsection{Systematic evolutionary analysis of SFTSV strain}

Full-length sequences of 49 SFTSV isolates from China, Japan, and South Korea from 2010 to 2016 were selected to cover the six known (A-F) genotypes of SFTSV. The maximum likelihood method was used for phylogenetic analysis. The results are shown in Figures $2 \mathrm{~A}, 2 \mathrm{~B}, 2 \mathrm{C}$. The results show that four SFTSV isolates in this study all clustered in genotype A (marked with in the figure), and no other genotypes were detected.

\subsection{Analysis of nucleotide and amino acid similarity of SFTSV strain}

The two SFTSV strains isolated from patients and the L, S segment sequence of SFTSV isolated from ticks in Dandong City, Liaoning Province and Yanji City, Jilin Province were 100\% identical, while the M segment sequence similarity was $99 \%$, which shows that the main source of transmission of SFTS in patients in the Liaoning Province are the ticks distributed in local or nearby areas.

Comparison of the four SFTSV strains with each genotype sequence revealed that it was the closest in nucleotide sequence and corresponding amino acid sequence to genotype A SFTSV (Table 2). The sequence similarities of the isolated L, M, and S nucleotides (and deduced amino acids) with other genetic A-type strains are between 95.95-100 (98.90-100), 97.12-100 (98.32-100), 95.08-100 (99.59-100)\%, respectively (Table $3-5)$. The nucleotide sequence similarity is above $95 \%$, and the corresponding amino acid similarity is above $98 \%$, which further suggest that the four SFTSV strains are genotype-A strains.

\subsection{Rickettsia molecular identification}

The 16S, glt $A$, omp $A$, and $o m p B$ conserved sequences carried by tick samples collected in this study were analyzed separately and found to be highly consistent with literature (Xu et al., 2019) (sequence similarities were $>99 \%$ ). Our results confirm that "Dandong-type Rickettsia ," which has the closest genetic sequence to Rickettsia in Heilongjiang, followed by Rickettsia in Japan, were carried by the ticks. These results show that "Dandong-type Rickettsia" is widely distributed in the Changbai Mountain forest area.

\subsection{Rickettsia-specific IgG antibody detection}


Seven serum samples were randomly selected for ELISA detection forRickettsia -specific IgG detection, including three for confirmed SFTS patient's serum (Dandong NO. 6; Donggang NO. 1; Fengcheng NO. 16); 4 patients with negative for SFTSV serum (Fengcheng NO. 7; Kaiyuan NO. 4; Haicheng NO. 14; Kuandian NO. 17), The results are shown in Table 1. Results show that sera from all 7 patients carried Rickettsia IgG (100\% positivity rate).

\section{Discussion}

Tick-borne diseases are the main cause of vector-borne diseases and have broad global distribution. They are mainly natural epidemic diseases, most common in forests, bushes, and semi-desert grasslands(Wu, Na, Wei, Zhu, \& Peng, 2013). There are at least 7 genera and 104 species of ticks in China. Northeast China has a mountainous terrain, has abundant biological resources, has geographical complexity, and has high species diversity, which provide an ecological and biological basis for the survival and reproduction of ticks. In this study, we found that the ticks distributed in the forest area of Northeast China are dominated by the long-horned blood ticks. Most patients affected by SFTS live in mountainous, hilly, or dense jungle areas and have a history of working outdoors. More than $80 \%$ of the cases are farmers, and there is history of field work, livestock contact, etc., and a few patients have history of tick bites. Among all the 17 suspected SFTS patients in this study, all cases occurred from the end of March to the end of June, and $94 \%$ of the cases (16/17) were from Changbai Mountain and other mountainous areas, forests, and hills in Northeast China, It is highly consistent with the active season and regional distribution of ticks in Liaoning Province.

SFTSV strains have been isolated from ticks in South Korea, Japan, and other regions sharing borders with or adjacent to China(Park et al., 2014; Yasukawa, 2008; Yun et al., 2015). Reports from the East Asian region show that the average mortality rate of SFTSV varies greatly, from $5.3 \%$ to $16.2 \%$ in China, $20 \%$ in Japan, and $23.3 \%$ in South Korea. Among the six known A-F genotypes of SFTSV(Fu et al., 2016), genotype B has the highest morbidity and mortality rates, which are significantly higher than those of the other genotypes. The incidence of the $\mathrm{F}$ genotype is lower than that of the B genotype, but its mortality rate is also higher, while the $\mathrm{A}$ genotype has the lowest mortality rate(Yun et al., 2020). The most common genotypes in China are genotypes $\mathrm{A}, \mathrm{D}$, and $\mathrm{F}$, and the mortality rate is relatively low, while the most common genotype in Japan and South Korea is B. Therefore, a comparison of viral genotypes and mortality indicates that the differences in reported case mortality may be related to the differences in the distribution of SFTSV genotypes in different countries. The number of clinical cases infected with certain SFTSV genotypes (especially reassortant genotypes) is small, which prevents the determination of the association between genotype and case mortality.

There is currently no vaccine or specific antiviral drug for SFTSV. Ribavirin is considered to be a potential antiviral drug for SFTS, but several retrospective studies have shown that ribavirin has not been effective in improving disease prognosis ( $\mathrm{Li}$ et al., 2018; Liu et al., 2013). (Li et al., 2019) found that benidipine hydrochloride (a calcium channel blocker, CCB) can inhibit SFTSV infection by interfering with virus internalization and reducing viral genome replication. Further experiments showed that a large number of CCBs including nifedipine inhibited SFTSV infection as well, indicating that CCB therapy may be developed as an effective strategy for the treatment of SFTSV infection.

Our previous research showed that among the various pathogens carried by ticks distributed in the Changbai Mountain forest area in China, theRickettsia positivity rate was the highest, and it was close to $10 \%$ in some areas (Qi et al., 2014). TheRickettsia detected in this study is consistent with the newly discovered "Dandong Rickettsia" genotype in 2018 and has is genetically close toRickettsia Heilongjiang. This genotype belongs to the class Alphaproteobacteria, order Rickettsiales, in the spotted fever group rickettsiae of the genus Rickettsia . The disease caused byRickettsia in Heilongjiang is called the "Far-East tick-borne Spotted Fever" (FESF), FESF has been considered as an important emerging infectious disease in Northeast Asia for this rickettsiosis has been diagnosed in Northeast of China, east-Siberian and far-eastern regions of Russia, and Japan. Rickettsia infection usually manifests as fever, headache, and rash, as well as muscle pain and arthralgia. The typical symptom is eschar-like skin lesions formed by the bite of a tick or mite, and the mortality rate is low under reasonable treatment. However, rashes and eschar did not appear in 
the 17 patients in this study, which may be because the skin lesions were hidden and painless, making them difficult to observe and easy to ignore. The diagnosis of rickettsial diseases can be achieved by RT-PCR or conventional PCR detection in diseased tissues (Denison, Amin, Nicholson, \& Paddock, 2014). However, sampling errors may cause false negatives, and patients with insignificant skin lesions may be completely ignored. Due to similar clinical manifestations caused by different rickettsial infections, there are crossreactions in serological methods. Pathogen culture is difficult, and the application of molecular techniques in whole blood are limited so far. Thus, rapid and reliable molecular blood detection of rickettsial diseases are still difficult to achieve. Indirect immunofluorescence assay is considered to be the gold standard, but due to its extensive cross-reactivity, this method has limited use in the determination of species within serogroups (Paris \& Dumler, 2016).

In our study, based on epidemiological data, clinical symptoms, and routine blood tests, 17 patients were initially classified as suspected SFTS patients. After molecular diagnostic testing, 5 SFTS patients were confirmed to have SFTSV. To understand whether there is a mixed infection of sftsv and rickettsia in patients bitten by ticks, From the 17 patient blood samples, 7 samples were chosen for Rickettsia -specific IgG antibody testing, and the positivity rate was $100 \%$. Of these 7 samples, 3 samples were from patients diagnosed with SFTS and 4 samples were from SFTS negative patients. Considering that ticks carry SFTSV and "Dandong-type Rickettsia ", we conclude that tick-borne SFTSV and "Dandong-type Rickettsia" infections are endemic in forest areas in Northeast China are more common. We hypothesize that SFTSV-"Dandongtype Rickettsia" coinfection complicates patient conditions. Therefore, we believe that in diagnosing tickborne diseases such as SFTSV, tick-borne encephalitis, and renal hemorrhagic fever syndrome, co-infection with Rickettsia will have to be considered. SFTS and other related tick-borne diseases should also be screened for patients with rickettsial infection.

\section{Acknowledgments}

We thank The Sixth People's Hospital of Shenyang for Providing the patient samples. This work was supported by the National Important Scientific \& Technology Project (2018ZX10101002-002). Title: The major vector-borne pathogens in the Belt and Road, Also got support from Education Department of Liaoning Province(LSNZD201606).

\section{Conflict of Interest}

The authors declare no competing interests.

\section{Ethics Statement}

The authors confirm that the ethical policies of the journal, as noted on the journal's author guidelines page, have been adhered to and the appropriate ethical review committee approval has been received. The 'Guidelines for Experimental Animals' of the Ministry of Science and Technology (Beijing, China) were followed. all participants involved in the study provided written informed consent.

\section{Data Availability Statement}

The data that support the findings of this study are available on request from the corresponding author. The data are not publicly available due to privacy or ethical restrictions.

\section{REFERENCES}

Adem, P.V. (2019). Emerging and re-emerging rickettsial infections.Seminars in diagnostic pathology, 36 , 146-151. https://doi.org/10.1053/j.semdp.2019.04.005

Denic, S., Janbeih, J., Nair, S., Conca, W., Tariq, W.U., \& Al-Salam, S. (2011). Acute Thrombocytopenia, Leucopenia, and Multiorgan Dysfunction: The First Case of SFTS Bunyavirus outside China. Case reports in infectious diseases, 2011 , 204056. https://doi.org/10.1155/2011/204056

Denison, A.M., Amin, B.D., Nicholson, W.L., \& Paddock, C.D. (2014). Detection of Rickettsia rickettsii, Rickettsia parkeri, and Rickettsia akari in skin biopsy specimens using a multiplex real-time polymerase 
chain reaction assay. Clinical infectious diseases : an official publication of the Infectious Diseases Society of America, 59 , 635-642. https://doi.org/10.1093/cid/ciu358

Fu, Y., Li, S., Zhang, Z., Man, S., Li, X., Zhang, W., .. (2016). Phylogeographic analysis of severe fever with thrombocytopenia syndrome virus from Zhoushan Islands, China: implication for transmission across the ocean. Scientific reports, 6 , 19563. https://doi.org/10.1038/srep19563

Li, H., Lu, Q.B., Xing, B., Zhang, S.F., Liu, K., Du J, .. (2018). Epidemiological and clinical features of laboratory-diagnosed severe fever with thrombocytopenia syndrome in China, 2011-17: a prospective observational study. The Lancet. Infectious diseases, 18 , 1127-1137. https://doi.org/10.1016/S14733099(18)30293-7

Li, H., Zhang, L.K., Li, S.F., Zhang, S.F., Wan, W.W., Zhang, Y.L., .. (2019). Calcium channel blockers reduce severe fever with thrombocytopenia syndrome virus (SFTSV) related fatality. Cell research, 29, 739-753. https://doi.org/10.1038/s41422-019-0214-z

Liu, H., Li, Q., Zhang, X., Li, Z., Wang, Z., Song, M., .. (2016). Characterization of rickettsiae in ticks in northeastern China.Parasites \& vectors, 9 , 498. https://doi.org/10.1186/s13071-016-1764-2

Liu, W., Lu, Q.B., Cui, N., Li, H., Wang, L.Y., Liu, K., .. (2013). Case-fatality ratio and effectiveness of ribavirin therapy among hospitalized patients in china who had severe fever with thrombocytopenia syndrome. Clinical infectious diseases : an official publication of the Infectious Diseases Society of America, 57, 1292-1299. https://doi.org/10.1093/cid/cit530

Liu, Y., Li, Q., Hu, W., Wu, J., Wang, Y., Mei, L., .. (2012). Person-to-person transmission of severe fever with thrombocytopenia syndrome virus. Vector borne and zoonotic diseases, 12 , 156-160. https://doi.org/10.1089/vbz.2011.0758

McMullan, L.K., Folk, S.M., Kelly, A.J., MacNeil, A., Goldsmith, C.S., Metcalfe, M.G., .. (2012). A new phlebovirus associated with severe febrile illness in Missouri. The New England journal of medicine, 367 , 834-841. https://doi.org/10.1056/NEJMoa1203378

Muehlenbachs, A., Fata, C.R., Lambert, A.J., Paddock, C.D., Velez, J.O., Blau, D.M., .. (2014). Heartland virus-associated death in tennessee.Clinical infectious diseases : an official publication of the Infectious Diseases Society of America, 59 , 845-850. https://doi.org/10.1093/cid/ciu434

Paris, D.H., \& Dumler, J.S. (2016). State of the art of diagnosis of rickettsial diseases: the use of blood specimens for diagnosis of scrub typhus, spotted fever group rickettsiosis, and murine typhus. Current opinion in infectious diseases, 29 , 433-439. https://doi.org/10.1097/QCO.0000000000000298

Park, S.W., Song, B.G., Shin, E.H., Yun, S.M., Han, M.G., Park, M.Y., .. (2014). Prevalence of severe fever with thrombocytopenia syndrome virus in Haemaphysalis longicornis ticks in South Korea. Ticks and tick-borne diseases, 5 , 975-977. https://doi.org/10.1016/j.ttbdis.2014.07.020

Parola, P., Paddock, C.D., \& Raoult, D. (2005). Tick-borne rickettsioses around the world: emerging diseases challenging old concepts. Clinical microbiology reviews, 18, 719-756. https://doi.org/10.1128/CMR.18.4.719-756.2005

Qi, Y., Gong, W., Xiong, X., Jiang, J., Wang, Y., Jiao, J., .. (2014). Microarray of surface-exposed proteins of Rickettsia heilongjiangensis for serodiagnosis of Far-eastern spotted fever. BMC infectious diseases, 14 , 332. https://doi.org/10.1186/1471-2334-14-332

Sun, Y., Guo, B., Yan, H., Wu, A.L., Yao, W.W., Chen, K., ... (2019). Patient with severe fever with thrombocytopenia syndrome virus infection and central nervous system disturbance in Dongyang, Zhejiang Province, China, 2017. Virology journal, 16 , 129. https://doi.org/10.1186/s12985-019-1230-9

Sun, Y., Qi, Y., Liu, C., Gao, W., Chen, P., Fu, L., .. (2014). Nonmuscle myosin heavy chain IIA is a critical factor contributing to the efficiency of early infection of severe fever with thrombocytopenia syndrome 
virus. Journal of virology, 88 , 237-248. https://doi.org/10.1128/JVI.02141-13

Takahashi, T., Maeda, K., Suzuki, T., Ishido, A., Shigeoka, T., Tominaga, T., ... (2014). The first identification and retrospective study of Severe Fever with Thrombocytopenia Syndrome in Japan. The Journal of infectious diseases, 209, 816-827. https://doi.org/10.1093/infdis/jit603

Wu, X.B., Na, R.H., Wei, S.S., Zhu, J.S., \& Peng, H.J. (2013). Distribution of tick-borne diseases in China. Parasites \& vectors, 6 , 119. https://doi.org/10.1186/1756-3305-6-119

Xu, H., Zhang, Q., Guan, H., Zhong, Y., Jiang, F., Chen, Z., \& Han, X. (2019). High Incidence of a Novel Rickettsia Genotype in Parasitic Haemaphysalis longicornis from China-North Korea Border.Scientific reports, 9 , 5373. https://doi.org/10.1038/s41598-019-41879-7

Yasukawa, M. (2008). [Human herpesvirus 6 in hematological diseases]. [Rinshō ketsueki] The Japanese journal of clinical hematology, $49,247-256$.

Yu, X.J., Liang, M.F., Zhang, S.Y., Liu, Y., Li, J.D., Sun, Y.L., .. (2011). Fever with thrombocytopenia associated with a novel bunyavirus in China. The New England journal of medicine, 364 , 1523-1532. https://doi.org/10.1056/NEJMoa1010095

Yun, S.M., Park, S.J., Kim, Y.I., Park, S.W., Yu, M.A., Kwon, H.I., .. (2020). Genetic and pathogenic diversity of severe fever with thrombocytopenia syndrome virus (SFTSV) in South Korea. JCI insight, 5 . https://doi.org/10.1172/jci.insight.129531

Yun, S.M., Song, B.G., Choi, W., Roh, J.Y., Lee, Y.J., Park, W.I., .. (2016). First Isolation of Severe Fever with Thrombocytopenia Syndrome Virus from Haemaphysalis longicornis Ticks Collected in Severe Fever with Thrombocytopenia Syndrome Outbreak Areas in the Republic of Korea. Vector borne and zoonotic diseases, 16 , 66-70. https://doi.org/10.1089/vbz.2015.1832

Yun, Y., Heo, S.T., Kim, G., Hewson, R., Kim, H., Park, D., .. (2015). Phylogenetic Analysis of Severe Fever with Thrombocytopenia Syndrome Virus in South Korea and Migratory Bird Routes Between China, South Korea, and Japan. The American journal of tropical medicine and hygiene, 93 , 468-474. https://doi.org/10.4269/ajtmh.15-0047

Zhang, X., Liu, Y., Zhao, L., Li, B., Yu, H., Wen, H., \& Yu, X.J. (2013). An emerging hemorrhagic fever in China caused by a novel bunyavirus SFTSV. Science China. Life sciences, 56 , 697-700. https://doi.org/10.1007/s11427-013-4518-9

\begin{tabular}{|c|c|c|c|c|c|c|c|}
\hline Characteristics & NO. $1^{\mathrm{a}}$ & NO. 2 & NO.3 & NO.4 & NO.5 & NO.6 & NO.7 \\
\hline \multicolumn{8}{|l|}{ Basic Information } \\
\hline Gender & Female & Male & Male & Male & Female & Male & Male \\
\hline Age & 47 & 44 & 54 & 56 & 76 & 41 & 58 \\
\hline Address & Donggang & Chang t $\mathrm{u}$ & Xi feng & Kaiyuan & An shan & Dandong & Fengcheng \\
\hline \multicolumn{8}{|l|}{ Etiological and Molecular biology Tests } \\
\hline PCR for SFTSV & Posi ${ }^{b}$ & $\mathrm{Neg}^{\mathrm{c}}$ & Neg & Neg & Neg & Posi & Neg \\
\hline Virus isolation & Posi & Neg & Neg & Neg & Neg & Posi & Neg \\
\hline $\begin{array}{l}\text { Rickettsia IgG antibody detection } \\
\text { Clinical }\end{array}$ & Posi & $\mathrm{NA}^{\mathrm{d}}$ & $\mathrm{NA}$ & Posi & $\mathrm{NA}$ & Posi & Posi \\
\hline & \multicolumn{7}{|c|}{ Clinical symptoms } \\
\hline Fever & {$[?]$} & {$[?]$} & {$[?]$} & [?] & {$[?]$} & {$[?]$} & [?] \\
\hline Headache & {$[?]$} & {$[?]$} & {$[?]$} & $x$ & {$[?]$} & {$[?]$} & {$[?]$} \\
\hline Unconsciousness & semicoma & $\times$ & $x$ & $x$ & $x$ & Delirium & $x$ \\
\hline Apathetic & {$[?]$} & $x$ & $x$ & $x$ & $\times$ & {$[?]$} & $x$ \\
\hline Muscle joint pain & {$[?]$} & {$[?]$} & {$[?]$} & [?] & {$[?]$} & ??] & {$[?]$} \\
\hline Nausea and vomiting & {$[?]$} & {$[?]$} & {$[?]$} & [?] & $x$ & ??] & $x$ \\
\hline Lymphadenopathy & NA & $x$ & $x$ & $x$ & $x$ & {$[?]$} & $x$ \\
\hline
\end{tabular}




\begin{tabular}{llllllll}
\hline Characteristics & NO. $1^{\mathrm{a}}$ & NO.2 & NO.3 & NO.4 & NO.5 & NO.6 & NO.7 \\
\hline $\begin{array}{l}\text { Pharyngeal congestion } \\
\text { Conjunctival congestion }\end{array}$ & {$[?]$} & {$[?]$} & {$[?]$} & {$[?]$} & $\times$ & $\times$ & {$[?]$} \\
Laboratory parameters(Normal range) & {$[?]$} & {$[?]$} & {$[?]$} & {$[?]$} & $\times$ & {$[?]$} & {$[?]$} \\
WBC $\left(4.0-10.0 \times 10^{9} / \mathrm{L}\right)$ & 5.6 & 7.2 & $2.2-$ & $3.2-$ & 6 & $1.2-$ & $2.3-$ \\
$\mathrm{PLT}\left(100-300 \times 10^{9} / \mathrm{L}\right)$ & $47-$ & $27-$ & $33-$ & $59-$ & 160 & $52-$ & $63-$ \\
$\mathrm{ALT}(0-40 \mathrm{U} / \mathrm{L})$ & $60-$ & $156-$ & $88-$ & $116-$ & 32 & $295-$ & 25 \\
$\mathrm{AST}(0-40 \mathrm{U} / \mathrm{L})$ & $268-$ & $320-$ & $228-$ & $175-$ & $50-$ & $688-$ & 35 \\
$\mathrm{BUN}(3.2-7.1 \mathrm{mmol} / \mathrm{L})$ & 5.2 & $9.5-$ & 4.74 & 4.59 & 5.02 & $7.4-$ & 4.7 \\
$\mathrm{CK}(25-200 \mathrm{U} / \mathrm{L})$ & $1026-$ & 140 & $356-$ & $991-$ & 51 & $4516-$ & $204-$ \\
$\mathrm{LDH}(135-225 \mathrm{U} / \mathrm{L})$ & $4032-$ & $100000-$ & $1976-$ & $1209-$ & $1293-$ & $3673-$ & $565-$ \\
\hline
\end{tabular}

Table 1. Basic Information, clinical symptoms, and laboratory parameters

a NO.1 was patient 201902845; NO.2 was patient 201903149; NO.3 was patient 201903151; NO.4 was patient 201901922; NO.5 was patient 201903976; NO.6 was patient 201904040;NO.7 was patient 201904339; NO.8 was patient 201904567; NO.9 was patient 201904687; NO.10 was patient 201904740; NO.11 was patient 201904737; NO.12 was patient 201905320;NO.13 was patient 201905311; NO.14 was patient 201905648; NO.15 was patient 201905649; NO.16 was patient 201906198; NO.17 was patient 201906189.

b Abbreviation of positive

c Abbreviation of negative

d No data

\begin{tabular}{|c|c|c|c|c|c|c|c|c|c|c|}
\hline $\begin{array}{l}\text { SFTSV } \\
\text { strain }\end{array}$ & $\begin{array}{l}\text { Source } \\
\text { of virus }\end{array}$ & $\begin{array}{l}\text { Geographi } \\
\text { origin }\end{array}$ & $\begin{array}{l}\text { calear of } \\
\text { isolation }\end{array}$ & $\begin{array}{l}\text { Genotype } \\
(\mathrm{L} / \mathrm{M} / \mathrm{S} \\
\text { segments) }\end{array}$ & $\begin{array}{l}\text { Sequence } \\
\text { identity } \\
(\%)\end{array}$ & $\begin{array}{l}\text { Sequence } \\
\text { identity } \\
(\%)\end{array}$ & $\begin{array}{l}\text { Sequence } \\
\text { identity } \\
(\%)\end{array}$ & $\begin{array}{l}\text { Sequence } \\
\text { identity } \\
(\%)\end{array}$ & $\begin{array}{l}\text { Sequence } \\
\text { identity } \\
(\%)\end{array}$ & $\begin{array}{l}\text { Sequence } \\
\text { identity } \\
(\%)\end{array}$ \\
\hline & & & & & $\begin{array}{l}\text { L } \\
\text { segments } \\
\text { Nucleotide }\end{array}$ & $\begin{array}{l}\mathrm{L} \\
\text { segments } \\
\text { Amino } \\
\text { acid }\end{array}$ & $\begin{array}{l}\text { M } \\
\text { segments } \\
\text { Nucleotide }\end{array}$ & $\begin{array}{l}\text { M } \\
\text { segments } \\
\text { Amino } \\
\text { acid }\end{array}$ & $\begin{array}{l}\text { S } \\
\text { segments } \\
\text { Nucleotide }\end{array}$ & $\begin{array}{l}\text { S } \\
\text { segments } \\
\text { Amino } \\
\text { acid }\end{array}$ \\
\hline \multicolumn{2}{|c|}{$\begin{array}{l}\text { LNHDD2011Guman } \\
9 \quad \text { serum }\end{array}$} & $\begin{array}{l}\text { Dandong } \\
\text { City, } \\
\text { Liaon- } \\
\text { ing } \\
\text { Province }\end{array}$ & 2019-9 & $\mathrm{A} / \mathrm{A} / \mathrm{A}$ & 99.98 & 100 & 99.08 & 99.63 & 100 & 100 \\
\hline \multicolumn{2}{|c|}{$\begin{array}{l}\text { LNHDG2019tuman } \\
6 \quad \text { serum }\end{array}$} & $\begin{array}{l}\text { Donggang } \\
\text { City, } \\
\text { Liaon- } \\
\text { ing } \\
\text { Province }\end{array}$ & 2019-6 & $\mathrm{A} / \mathrm{A} / \mathrm{A}$ & 99.98 & 100 & 99.08 & 99.63 & 100 & 100 \\
\hline $\begin{array}{l}\text { LNTEDG } \\
6\end{array}$ & $\begin{array}{l}\text { 20H@emaph? } \\
\text { longi- } \\
\text { cornis }\end{array}$ & $\begin{array}{l}\text { stahidaogou, } \\
\text { Dan- } \\
\text { dong } \\
\text { City } \\
\text { Liaon- } \\
\text { ing } \\
\text { province }\end{array}$ & 2019-6 & $\mathrm{A} / \mathrm{A} / \mathrm{A}$ & 99.98 & 100 & 99.08 & 99.63 & 100 & 100 \\
\hline
\end{tabular}




\begin{tabular}{|c|c|c|c|c|c|c|c|c|c|c|}
\hline $\begin{array}{l}\text { SFTSV } \\
\text { strain }\end{array}$ & $\begin{array}{l}\text { Source } \\
\text { of virus }\end{array}$ & $\begin{array}{l}\text { Geographic } \\
\text { origin }\end{array}$ & $\begin{array}{l}\text { calear of } \\
\text { isolation }\end{array}$ & $\begin{array}{l}\text { Genotype } \\
(\mathrm{L} / \mathrm{M} / \mathrm{S} \\
\text { segments })\end{array}$ & $\begin{array}{l}\text { Sequence } \\
\text { identity } \\
(\%)\end{array}$ & $\begin{array}{l}\text { Sequence } \\
\text { identity } \\
(\%)\end{array}$ & $\begin{array}{l}\text { Sequence } \\
\text { identity } \\
(\%)\end{array}$ & $\begin{array}{l}\text { Sequence } \\
\text { identity } \\
(\%)\end{array}$ & $\begin{array}{l}\text { Sequence } \\
\text { identity } \\
(\%)\end{array}$ & $\begin{array}{l}\text { Sequence } \\
\text { identity } \\
(\%)\end{array}$ \\
\hline $\begin{array}{l}\text { LNTYJ2 } \\
6\end{array}$ & $\begin{array}{c}\text { 19aemap } \\
\text { longi- } \\
\text { cornis }\end{array}$ & $\begin{array}{l}\text { sdlasiji } \\
\text { City, } \\
\text { Jilin } \\
\text { Province }\end{array}$ & $2019-6$ & $\mathrm{~A} / \mathrm{A} / \mathrm{A}$ & 99.98 & 100 & 99.08 & 99.63 & 100 & 100 \\
\hline
\end{tabular}

Table 2. Sequence information for SFTSV isolates in this study

\begin{tabular}{llllllllllllll}
\hline Strain & & 1 & 2 & 3 & 4 & 5 & 6 & 7 & 8 & 9 & 10 & 11 & 12 \\
\hline LNHDD2019-9 & 1 & & 100 & 100 & 100 & 98.30 & 99.09 & 98.63 & 97.82 & 97.83 & 97.86 & 97.94 & 97.86 \\
LNTYJ2019-6 & 2 & 100 & & 100 & 100 & 98.30 & 99.09 & 98.63 & 97.82 & 97.83 & 97.86 & 97.94 & 97.86 \\
LNTEDG2019-6 & 3 & 100 & 100 & & 100 & 98.30 & 99.09 & 98.63 & 97.82 & 97.83 & 97.86 & 97.94 & 97.86 \\
LNHDG2019-6 & 4 & 100 & 100 & 100 & & 98.30 & 99.09 & 98.63 & 97.82 & 97.83 & 97.86 & 97.94 & 97.86 \\
SFTSV & 5 & 99.18 & 99.18 & 99.18 & 99.18 & & 98.23 & 97.95 & 98.08 & 98.65 & 98.72 & 98.89 & 98.04 \\
JS2014-18 & 6 & 99.71 & 99.71 & 99.71 & 99.71 & 98.99 & & 98.83 & 98.98 & 98.08 & 98.11 & 98.15 & 98.04 \\
ZJZHSH-WSH & 7 & 99.81 & 99.81 & 99.81 & 99.81 & 98.99 & 99.71 & & 99.15 & 97.70 & 97.73 & 97.78 & 97.73 \\
CB2 & 8 & 99.86 & 99.86 & 99.86 & 99.86 & 99.04 & 99.76 & 99.86 & & 97.82 & 97.86 & 97.90 & 97.82 \\
JS3 & 9 & 99.47 & 99.47 & 99.47 & 99.47 & 99.04 & 99.47 & 99.47 & 99.52 & & 99.69 & 99.61 & 98.47 \\
JS24 & 10 & 99.47 & 99.47 & 99.47 & 99.47 & 99.04 & 99.47 & 99.47 & 99.52 & 99.81 & & 99.67 & 98.50 \\
JS2013-71 & 11 & 99.52 & 99.52 & 99.52 & 99.52 & 99.09 & 99.52 & 99.52 & 99.57 & 99.86 & 99.86 & & 98.54 \\
LN3 & 12 & 99.57 & 99.57 & 99.57 & 99.57 & 98.94 & 99.47 & 99.57 & 99.62 & 99.52 & 99.52 & 99.57 & \\
JS2014-Hedgehog-01 & 13 & 99.47 & 99.47 & 99.47 & 99.47 & 98.94 & 99.47 & 99.47 & 99.52 & 99.52 & 99.52 & 99.57 & 99.62 \\
HL/Adult/G2 & 14 & 99.62 & 99.62 & 99.62 & 99.62 & 98.99 & 99.52 & 99.62 & 99.66 & 99.87 & 99.87 & 99.88 & 99.89 \\
2011YSC60 & 15 & 99.52 & 99.52 & 99.52 & 99.52 & 98.90 & 99.42 & 99.52 & 99.57 & 99.47 & 99.47 & 99.52 & 99.57 \\
2012YXX1 & 16 & 99.62 & 99.62 & 99.62 & 99.62 & 98.99 & 99.52 & 99.62 & 99.66 & 99.87 & 99.87 & 99.88 & 99.89 \\
HB2016-047 & 17 & 99.62 & 99.62 & 99.62 & 99.62 & 98.99 & 99.52 & 99.62 & 99.66 & 99.87 & 99.87 & 99.88 & 99.89 \\
YSC3 & 18 & 99.66 & 99.66 & 99.66 & 99.66 & 99.04 & 99.47 & 99.57 & 99.62 & 99.52 & 99.52 & 99.57 & 99.52 \\
\hline
\end{tabular}

Table 3. Comparison (\% similarity) of A-genotype SFTSV L segment nucleotide (top right) and amino acid (bottom left) sequences

Table 4. Comparison (\% similarity) of A-genotype SFTSV M segment nucleotide (top right) and amino acid (bottom left) sequences

\begin{tabular}{llllllllllllll}
\hline Strain & & 1 & 2 & 3 & 4 & 5 & 6 & 7 & 8 & 9 & 10 & 11 & 12 \\
\hline LNHDG2019-6 & 1 & & 99.97 & 99.97 & 99.94 & 99.06 & 98.74 & 98.30 & 97.82 & 97.91 & 97.97 & 97.88 & 98.03 \\
LNTYJ2019-6 & 2 & 100 & & 100 & 99.97 & 99.09 & 98.77 & 98.32 & 97.85 & 97.94 & 98.00 & 97.91 & 98.06 \\
LNTEDG2019-6 & 3 & 100 & 100 & & 99.97 & 99.09 & 98.77 & 98.32 & 97.85 & 97.94 & 98.00 & 97.91 & 98.06 \\
LNHDD2019-9 & 4 & 100 & 100 & 100 & & 99.06 & 98.74 & 98.30 & 97.82 & 97.91 & 97.97 & 97.88 & 98.03 \\
JS2014-18 & 5 & 99.53 & 99.53 & 99.53 & 99.53 & & 98.79 & 98.35 & 97.62 & 97.91 & 97.97 & 97.97 & 98.12 \\
CB2 & 6 & 99.53 & 99.53 & 99.53 & 99.53 & 99.72 & & 98.56 & 97.38 & 97.74 & 97.79 & 97.74 & 97.88 \\
ZJZHSH-WSH & 7 & 99.07 & 99.07 & 99.07 & 99.07 & 99.25 & 99.35 & & 97.18 & 97.47 & 97.53 & 97.59 & 97.62 \\
SFTSV & 8 & 98.88 & 98.88 & 98.88 & 98.88 & 98.79 & 98.79 & 98.32 & & 97.79 & 97.85 & 97.85 & 97.88 \\
JS24 & 9 & 99.07 & 99.07 & 99.07 & 99.07 & 99.35 & 99.25 & 98.79 & 98.51 & & 99.76 & 99.59 & 99.15 \\
JS3 & 10 & 99.25 & 99.25 & 99.25 & 99.25 & 99.53 & 99.44 & 98.97 & 98.70 & 99.81 & & 99.65 & 99.21 \\
JS2013-71 & 11 & 99.25 & 99.25 & 99.25 & 99.25 & 99.53 & 99.44 & 98.97 & 98.70 & 99.63 & 99.81 & & 99.15
\end{tabular}




\begin{tabular}{|c|c|c|c|c|c|c|c|c|c|c|c|c|c|}
\hline Strain & & 1 & 2 & 3 & 4 & 5 & 6 & 7 & 8 & 9 & 10 & 11 & 12 \\
\hline LN3 & 12 & 99.25 & 99.25 & 99.25 & 99.25 & 99.53 & 99.44 & 98.97 & 98.70 & 99.63 & 99.81 & 99.81 & \\
\hline JS2014-Hedgehog-01 & 13 & 99.16 & 99.16 & 99.16 & 99.16 & 99.44 & 99.35 & 98.88 & 98.60 & 99.25 & 99.44 & 99.44 & 99.44 \\
\hline HL/Adult/G2 & 14 & 99.16 & 99.16 & 99.16 & 99.16 & 99.44 & 99.35 & 98.88 & 98.79 & 99.35 & 99.53 & 99.53 & 99.53 \\
\hline 2012YXX1 & 15 & 99.25 & 99.25 & 99.25 & 99.25 & 99.53 & 99.44 & 98.97 & 98.88 & 99.44 & 99.63 & 99.63 & 99.63 \\
\hline 2011YPQ12 & 16 & 98.70 & 98.70 & 98.70 & 98.70 & 98.97 & 98.88 & 98.42 & 98.32 & 98.88 & 99.07 & 99.25 & 99.07 \\
\hline HB2016-047 & 17 & 98.88 & 98.88 & 98.88 & 98.88 & 99.16 & 99.07 & 98.60 & 98.51 & 99.07 & 99.29 & 99.44 & 99.25 \\
\hline
\end{tabular}

\begin{tabular}{llllllllllllll}
\hline Strain & & 1 & 2 & 3 & 4 & 5 & 6 & 7 & 8 & 9 & 10 & 11 & 12 \\
\hline LNHDD2019-9 & 1 & & 100 & 100 & 100 & 99.32 & 98.64 & 97.06 & 98.36 & 97.34 & 97.51 & 97.29 & 97.57 \\
LNTYJ2019-6 & 2 & 100 & & 100 & 100 & 99.32 & 98.64 & 97.06 & 98.36 & 97.34 & 97.51 & 97.29 & 97.57 \\
LNTEDG2019-6 & 3 & 100 & 100 & & 100 & 99.32 & 98.64 & 97.06 & 98.36 & 97.34 & 97.51 & 97.29 & 97.57 \\
LNHDG2019-6 & 4 & 100 & 100 & 100 & & 99.32 & 98.64 & 97.06 & 98.36 & 97.34 & 97.51 & 97.29 & 97.57 \\
SFTSV & 5 & 100 & 100 & 100 & 100 & & 98.81 & 97.17 & 98.25 & 97.63 & 97.80 & 97.57 & 97.85 \\
JS2014-18 & 6 & 100 & 100 & 100 & 100 & 100 & & 97.57 & 98.19 & 97.91 & 98.08 & 97.85 & 98.13 \\
ZJZHSH-WSH & 7 & 100 & 100 & 100 & 100 & 100 & 100 & & 97.18 & 96.27 & 96.44 & 96.33 & 96.50 \\
CB2 & 8 & 100 & 100 & 100 & 100 & 100 & 100 & 100 & & 96.89 & 97.06 & 96.89 & 97.12 \\
JS3 & 9 & 99.59 & 99.59 & 99.59 & 99.59 & 99.59 & 99.59 & 99.59 & 99.59 & & 99.83 & 99.60 & 99.38 \\
JS24 & 10 & 100 & 100 & 100 & 100 & 100 & 100 & 100 & 100 & 99.59 & & 99.77 & 99.55 \\
JS2013-71 & 11 & 100 & 100 & 100 & 100 & 100 & 100 & 100 & 100 & 99.59 & 100 & & 99.32 \\
LN3 & 12 & 100 & 100 & 100 & 100 & 100 & 100 & 100 & 100 & 99.59 & 100 & 100 & \\
JS2014-Hedgehog-01 & 13 & 100 & 100 & 100 & 100 & 100 & 100 & 100 & 100 & 99.59 & 100 & 100 & 100 \\
HL/Adult/G2 & 14 & 100 & 100 & 100 & 100 & 100 & 100 & 100 & 100 & 99.59 & 100 & 100 & 100 \\
2011YSC60 & 15 & 100 & 100 & 100 & 100 & 100 & 100 & 100 & 100 & 99.59 & 100 & 100 & 100 \\
2012YXX1 & 16 & 100 & 100 & 100 & 100 & 100 & 100 & 100 & 100 & 99.59 & 100 & 100 & 100 \\
HB2016-047 & 17 & 100 & 100 & 100 & 100 & 100 & 100 & 100 & 100 & 99.59 & 100 & 100 & 100 \\
YSC3 & 18 & 100 & 100 & 100 & 100 & 100 & 100 & 100 & 100 & 99.59 & 100 & 100 & 100 \\
\hline
\end{tabular}

Table 5. Comparison (\% similarity) of A-genotype S segment nucleotide (top right) and amino acid (bottom left) sequences

Figure 1. Regional distribution of reported cases and tick isolation in Liaoning Province in 2019. Areas 1-6 are where the 17 patients live; tick samples were collected in areas 1-7; SFTSV was isolated from ticks collected in areas 4 and 7 .

Figure 2A. Phylogenetic analysis based on the complete nucleotide sequences of the L segment of SFTSV isolates.

Figure 2B. Phylogenetic analysis based on the complete nucleotide sequences of the M segment of SFTSV isolates.

Figure 2C. Phylogenetic analysis based on the complete nucleotide sequences of the S segment of SFTSV isolates.

\section{Hosted file}

Figure.pptx available at https://authorea.com/users/360146/articles/481853-coinfection-ofsftsv-genotype-a-with-rickettsia-among-farmers-in-northeast 